DOI: http://dx.doi.org/10.18524/1810-4215.2018.31.144435

\title{
ZELDOVICH LOCAL PANCAKE: DARK ENERGY DOMINATION
}

\author{
A. D. Chernin ${ }^{1}$, I. D. Karachentsev ${ }^{2}$, N. V. Emelyanov ${ }^{1}$ \\ ${ }^{1}$ Sternberg Astronomical Institute, Moscow University, Moscow, Russia \\ Arthur.Cherninl@gmail.com, \\ ${ }^{2}$ Special Astrophysical Observatory, Russian Academy of Sciences, Nizhniy Arkhys, Russia
}

\begin{abstract}
Zeldovich Local Pancake is a twodimensional system of 15 giant galaxies nearest to us. Two of the galaxies, the Milky Way and the Andromeda Galaxy, move to each other in the Local Group, while the rest of the galaxies are located around the group at the distances up to $10 \mathrm{Mpc}$ from the group barycenter and move away from it forming a local expansion outflow. We use recent Hubble Space Telescope data on local giants and their numerous fainter companions to study the dynamical structure and evolutionary trends of the expanding system. N-body computer model, which reproduces the observed kinematics of the flow, is constructed under the assumption that the system as a whole is embedded in the universal dark energy background. In the model, the motions of the flow bodies are controlled by their mutual attraction force and the repulsion force produced by the dark energy. It is found that the repulsion dominates the force field of the system. Because of this, the the system expands with acceleration. The dark energy domination increases with time and introduces to the expansion flow an asymptotically linear velocity-distance relation with the universal time-rate (the Hubble constant) that depends on the dark energy density only.
\end{abstract}

Key words: galaxies: groups: general, galaxies: kinematics and dynamics, dark energy.

АБСТРАКТ. Локальний млинець Зельдовича двовимірна система, яка утворена 15 найближчими до нас гігантськими галактиками. Дві з них, Чумацький Шлях i галактика в Андромеді, рухаються назустріч один одному в Місцевій Групі галактик, тоді як інші галактики знаходяться навколо групи на відстанях до 10 Мпс від ㄲï барицентра і рухаються геть від неї, утворюючи місцевий потік розбігання. Ми спираємося на свіжі дані спостережень космічного келескопа “ Хаббл " для місцевих гігантських галактик i ïx чисельних і менших за розмірами компаньйонів, для вивчення сучасної динамічної структури потоку розбігання і тенденцій його еволюції. Ми будуємо також чисельну модель задачі декількох тел, яка відтворює спостережувану кинематику i динаміку потоку в припущенні, що система як ціле занурена в універсальний фон темної енергії. У цій моделі рух тел потоку управляється силою ïх взаємного тяжіння i силою відштовхування, яка створюється темною енергією. Встановлено, що сила відштовхування домінує в силовому полі системи. 3 цієї причини розширення системи відбувається 3 прискоренням. Домінування темної енергії посилюється з часом і асимптотично привносить в систему лінійну залежність швидкості від відстані з універсальним коефіцієнтом (постійної Хаббла), що залежать тільки від щільності темної енергіï.

Ключові слова: галактики, групи галактик, кінематика і динаміка галактик, темна енергія.

\section{Introduction}

A half century ago, the notion of cosmic pancakes was introduced by Ya. B. Zeldovich in his famous theory of galaxy formation (Zeldovich, 1970). Initially, pancakes were considered as flattened clouds of protogalactic gas produced by gravitational instability in the early Universe. Now the pancake concept is treated in a wider context with not only gasdynamical processes, but also motions of two-dimensional (2D) cosmic Nbody systems which is applicable to nonlinear stages of gravitational instability (Chernin et al., 2015 and references therein). It is important that such $2 \mathrm{D}$ systems are really observed in the Cosmic Web on the spatial scales from several to hundreds megaparsec (Mpc). The nearest to the Milky Way 2D system is the N-body ensemble of 15 giant galaxies observed at distances up to $10 \mathrm{Mpc}$ from the barycentre of the Local Group. Following the suggestion by one of us (I.K.), we refer to this system as Zeldovich Local Pancake (ZLP). The 
ZLP galaxies and their numerous fainter companions have recently been observed (Karachentsev \& Kudrya, 2013; Karachentsev et al., 2013, 2014) with the Hubble Space Telescope (HST). Two of the local giants of the ensemble, which are the Milky Way and the Andromeda Nebula, are moving towards each other inside the Local Group, while a dozen others are moving away from the group with radial velocities from 100 to 900 $\mathrm{km} / \mathrm{s}$. Each of the ZLP members contributes more or less equally to the system's total matter mass which is estimated as $10^{14}$ solar masses.

The physics behind the observed properties of the systems is also enriched now compared to the original pancake dynamics: it includes both gravity of matter and anti-gravity produced by universal dark energy. All the cosmic systems and the Cosmic Web as a whole are embedded in the dark energy background. We adopt that the dark energy is represented by Einstein's cosmological constant $\Lambda$, as in the currently standard $\Lambda \mathrm{CDM}$ cosmology. The constant is positive, which implies that the dark energy produces a repulsive force of anti-gravity. The cosmic repulsion acts not only on the global cosmological distances where it was originally discovered in observations (Riess et al., 1998; Perlmutter et al., 1999), but actually everywhere in space. We will use the works on the local dark energy effects (Chernin, 2001) to show below that in the volume of the observed Local Group ( $R 1 \mathrm{Mpc})$, the dark energy anti-gravity is weaker than the gravity produced by the matter mass of the group; but the anti-gravity repulsion (described in the reference frame of the group barycentre) dominates at distances $>13 \mathrm{Mpc}$ from group's barycentre. The anti-gravity domination makes the ZLP expand with acceleration. Because of this, the ZLP is cooling with time and its time-rate ("the Hubble constant") $H(t)$ tends to the current cosmological value $H_{0}=70 \mathrm{~km} /(\mathrm{sMpc})$.

We describe the motions of the ZLP giants with the use of the N-body computer model in combination with the HST data (Karachentsev et al., 2013, 2014; Karachentsev, Kaisina \& Makarov, 2014). The antigravity of dark energy effects at the present epoch, as well as in the future, are in the focus of our discussion here. We do not discuss, however, the origin and early evolution of the ZLP in its past. The observed present-day velocities and distances of the giants are used as initial conditions in our model.

\section{ZLP: Basic HST data}

The observation data on the local giant galaxies and their companions are presented in the recently published Updated Nearby Galaxy Catalogue (UNGC) by Karachentsev et al. (2013); see also Karachentsev \& Kudrya (2014) and Karachentsev et al. (2014). The catalogue contains systematic and homogeneous data on coordinates, distances, radial velocities and other basic physical parameters of about 800 galaxies at distances up to $10 \mathrm{Mpc}$. More than $300 \mathrm{UNGC}$ galaxies at these distances have been observed with the HST for about $330 \mathrm{HST}$ orbits. The unique resolution available in the HST observations allowed us to use the Tip of the Red Giant Branch (TRGB) method for precise measurements of distances to more than 300 nearby galaxies with an accuracy from 10 to 30 per cent. Precise data on the radial velocities of the galaxies have also been compiled in the UNGC.

According to Karachentsev et al. (2013, 2014), there are 15 nearby giant galaxies at distances up to $10 \mathrm{Mpc}$ : they are the Milky Way and the Andromeda Nebula (gravitationally bound in the Local Group), and galaxies M81, NGC 5128, IC342, NGC 253, NGC 4736, NGC 5236, NGC 6946, M101, NGC 4258, NGC 4594, NGC 3115, NGC 3627 and NGC 3368. Each of the giants of the ZLP is actually the main galaxy of a group (similar to the Local Group), which includes the galaxy itself and its extended dark matter halo together with companion galaxies therein. The mass $M$ of the system is the total orbital mass of the group. The UNGC data are used to estimate the masses of the groups via motions of their 351 less-massive companions (Karachentsev \& Kudrya, 2014). The distance $R$ of a giant and its radial velocity $V$ are calculated relative to the barycentre of the Local Group.

The nearest to the Local Group is M81 galaxy with a distance of $3.6 \mathrm{Mpc}$. Its recession velocity (relative to the Local Group barycentre) is about $100 \mathrm{~km} / \mathrm{s}$, the lowest in the ZLP. The mass of the galaxy is $5 \times 10^{12}$ solar masses (we give here rounded numbers, the exact figures with error bars may be found in the paper by Karachentsev \& Kudrya, 2014). The most distant from us is the galaxy NGC 3368 at $10.4 \mathrm{Mpc}$ with the radial velocity $740 \mathrm{~km} / \mathrm{s}$ which is the second largest velocity in the ZLP. Its mass is $2 \times 10^{13}$ solar masses, which is the second largest mass in the ZLP. The most massive galaxy is probably NGC 4594 at $9.3 \mathrm{Mpc}$; its mass is $3 \times 10^{13}$ solar masses, but with an error of $2 \times 10^{13}$ solar masses, the largest error in the data. The major gross parameter of the ZLP is its total mass $M_{t o t}=8 \times 10^{13}$ solar masses, which is the sum of the matter (dark matter and baryons) masses of the ZLP member. 2014).

\section{ZLP: N-body model}

The structure and evolution of the ZLP may be studied with a computer model which treats it as a twodimensional (2D) gravitational N-body system embedded in the dark energy background. This is a nonrelativistic isolated conservative system of point-like masses interacting with each other via Newton's mutual gravity and undergoing Einstein's anti-gravity pro- 
duced by the universal - dark energy background. Dark energy is considered as continuous medium with the density $\rho_{\Lambda}=c^{2} \Lambda /(8 \pi G)$, which is positive and constant in space and time in any reference frame (here $G$ is the Newtonian gravitational constant and $c$ is the speed of light). The currently adopted value of the universal dark energy density is $\rho_{\Lambda}=0.7 \times 10^{29} \mathrm{~g} / \mathrm{cm}^{3}$. The dynamical effects of dark energy in the model are described by relations that come from General Relativity with Einstein non-zero cosmological constant $\Lambda$ and the currently standard $\Lambda \mathrm{CDM}$ cosmology (see for detailes Chernin, 2001; Byrd et al., 2012). As macroscopic medium, dark energy is characterized by the equation of state

$$
p_{\Lambda}=-\rho_{\Lambda} c^{2},
$$

where $p_{\Lambda}$ is the dark energy pressure. Dark energy cannot serve as a reference frame, and it is co-moving to any like trivial emptiness.

In General Relativity, the effective gravitating density is determined by both density and pressure of any medium:

$$
\rho_{e f f}=\rho_{\Lambda}+3 p_{\Lambda} c^{2} .
$$

For dark energy, the effective density, $\rho_{\text {eff }}=$ $-2 \rho_{\Lambda}<0$, is negative, and because of this dark energy produces the repulsion, or anti-gravity. General Relativity indicates also that the passive gravitational density is the sum $\rho_{\text {pass }}=\rho+p / c^{2}$ for any fluid. The value $\rho_{\text {pass }}$ is zero for dark energy. According to the equivalence principle, the passive gravitational mass is equal to the inertial density. Thus, the inertial density of dark energy is also zero. This implies that dark energy is affected neither by the external gravity of matter nor by its own anti-gravity.

In the ZLP, the local dynamical effects of dark energy are treated in terms of Newtonian mechanics; this is possible because the velocities of the local galaxies are small compared to the speed of light, and the spatial differences in both gravity and anti-gravity potentials are much smaller (in absolute value) compared to the speed of light squared. According to this weak field approximation, Einstein's law of anti-gravitation states that any body in the Universe is affected by the repulsive force, which is proportional to the dark energy density $\rho_{\Lambda}$ and to the distance $R$ of the body from the origin of the adopted reference frame:

$$
F_{\Lambda}=8 \pi / 3 G \rho_{\Lambda} R \text {. }
$$

This force acts along the direction from the origin, and it gives the force per unit mass of the body (i.e. acceleration) in the projection on the body radius-vector. This relation can be rewritten in the form

$$
F_{\Lambda}=H_{\Lambda}^{2} \Lambda R
$$

where $H_{\Lambda}=\left(8 \pi / 3 G \rho_{\Lambda}\right)^{1 / 2}=61 k m /(s M p c)$ is the cosmic universal Hubble constant, which is valid on both global and local spatial scales everywhere in space and at any moment of time.

The equations of motion for the ZLP bodies are based on the relations (3) and (4) with the initial conditions specified at the present moment of cosmic time $t=t_{0}=13.7 \mathrm{Myr}$. These are the observed positions of the flow bodies and their measured radial velocities recalculated to the barycentre of the Local Group. The model has strong limitations: (i) it is valid only when and where the distances between the system bodies (giant galaxies with their dark matter haloes) are large enough compared to the sizes of the bodies; (ii) it does not describe the origin and early evolution of the flow. As it may be seen from the data above, the first limitation is well satisfied for the observed present-day state of the flow; it is clearly satisfied as well in the future states of the system. The second limitation comes from the first one and also from the account of complex physical processes responsible for the formation and evolution of the local systems in their early history. Observations provide us with the radial velocities of the galaxies, but say nothing about their tangential (transverse) velocities. This is an obvious flaw in any dynamical model of extragalactic astronomy where the full velocity vector is needed for correct formulation of initial conditions. Fortunately, the coordinate origin of our model is located close to the barycentre of the ZLP, so that most measured velocities are actually radial. Having this in mind, we assume that the transversal velocities are zero in the initial conditions for the model.

The numerical integration of equations of motions for the ZLP bodies are made with the use of the standard computer method with the automatic choice of the integration step. The result reproduces the motions of the ZLP bodies from the Local Group barycentre as functions of time from $t=13$ Gyr to $t=25 \mathrm{Gyr}$. The major features of the state and evolution of the system are proving to be as follows:

1) the ZLP bodies are moving away from the initial position, so that the system as a whole is expanding with time;

2) the recession velocities of the ZLP bodies are growing with time, which indicates that the expansion of the system proceeds with acceleration;

3) the accelerating expansion means that the dark energy anti-gravity is stronger than the mutual gravity attraction of the ZLP bodies;

4) the domination of the dark energy anti-gravity in the ZLP dynamics is similar to the cosmological effect of the global accelerating expansion at the present-day state of the whole Universe.

$5)$ with their growth with time, the ZLP trajectories converge to the straight line $V=H_{\Lambda} R$ going from the coordinate origin in the $(V$ to $R$ ) phase space;

$6)$ accordingly, the mean radial velocity dispersion decreases in ZLP with the trajectories growth, and the 
system becomes increasingly regular and "cold".

As we may see now, the dark energy domination is the major factor of the ZLP state and dynamical evolution at $t>t_{0}$. The dark energy anti-gravity becomes stronger, while the mutual gravity of the galaxies vanishes with the system expansion. These findings have become possible due to a recent combination of the HST observational data (Karachentsev et al., 2013, 2014; Karachentsev \& Kudrya, 2014) with a computer model for the ZLP.

\section{Conclusions}

The ZLP and some other known local expanding systems and flows are observed at advanced stages of their evolution when they have already reached a certain degree of regularity. At these stages, the anti-gravity domination became the key factor of the system evolution. Earlier in the cosmic times, the dynamical effects of the dark energy were insignificantly weak and the cosmic structures of various spatial scales formed due to gravitational collapse driven mostly by dark matter. These evolutionary stages were studied by Zeldovich in 1970. It would be interesting to study also the transition epoch when the dark energy anti-gravity becomes strong enough to terminate collapse. That kind of gravity vs. anti-gravity interplay may be one of the important aspects of general theory of cosmic structure formation.
Acknowledgements. We are grateful to G. Bisnovatyi-Kogan, G. Byrd, Yu. Efremov, M. Pruzhinskaya, P. Teerikorpi, M. Valtonen and A. Zasov for helpful discussions.

\section{References}

Byrd G.G., Chernin A.D., Teerikorpi P., Valtonen M.J.: 2012 Paths to Dark Energy Berlin/Boston De Gruyter

Chernin A.D.: 2001, Phys.-Usp., 44, 1099.

Chernin A.D., Emelyanov N.V., Karachentsev I.D.: 2015, MNRAS, 449, 2069.

Karachentsev I.D., Makarov D.I. Kaisina E.I.: 2013 , $A J, \mathbf{1 4 5}, 101$.

Karachentsev I.D., Kudrya Yu.N.: 2014, AJ, 148, 50. Karachentsev I.D., Kaisina E.I., Makarov D.I.: 2014, $A J$, 147, 13 .

Perlmutter S. et al.: 1999, ApJ, 517, 565.

Riess A.G. et al.: 1998, AJ, 116, 1009.

Zeldovich Ya.B.: 1970, $A \mathscr{E} A, \mathbf{5}, 84$. 